\title{
Effects of anti-TNF therapy on ophtalmological complications in children with rheumatic diseases
}

\author{
Marijan Frkovic ${ }^{*}$, Nenad Vukojevic ${ }^{2}$, Marija Jelusic ${ }^{1}$ \\ From 21st European Pediatric Rheumatology (PReS) Congress \\ Belgrade, Serbia. 17-21 September 2014
}

\section{Introduction}

Biologics started a new era in treatment of different aspects of rheumatic disases. Authors present the effects of anti-TNF therapy on ophtalmological complications in their patients with juvenile idiopathic artritis (JIA), juvenile dermatomiositis (JDM) and sarcoidosis.

\section{Objectives}

To investigate therapeutical sucess of anti-TNF therapy on ophtalmological complications in children with rheumatic diseases.

\section{Methods}

Retrospective chart rewiev of all rheumatic patients with ophtalmological complications treated with anti-TNF therapy at Division of Paediatric Rheumatology and Immunology, University Hospital Centre Zagreb, during 2009. - 2013. period.

\section{Results}

Among 54 children treated with anti-TNF therapy at our Division during 2009. - 2013. period, 10 were detected with ophtalmologycal complications (8 girls, 2 boys), 8 with JIA, 1 with JDM and 1 with sarcoidosis. Nine patients had chronic uveitis (JIA, sarcoidosis) and 1 had ...cotton wool" retinal lesions accompanied with bilateral papilar oedema (JDM). Avarage time between the begining of disease and start of anti-TNF therapy was 2.9 years ( 28 days -9 years). Initial anti-TNF therapy was adalimumab in 5 cases (4 JIA, 1 sarcoidosis), etanercept in 4 cases (JIA) and infliximab in 1 case (JDM). During therapy, etanercept was changed to adalimumab in 2 cases, due to inefficacy on uveitis. Adalimumab was partially effective in 1 case (sarcoidosis). Infliximab, applied in patient with JDM and ...cotton wool" retinal lesions accompanied with bilateral papilar oedema, led to fast and complete recovery of eye changes and other aspects of the disease.

\section{Conclusion}

Anti-TNF therapy was successfull in $90 \%$ of our cases. To our opinion, anti-TNF therapy should be early introduced in rheumatic patients with severe ophtalmological complications.

\section{Disclosure of interest}

None declared.

\section{Authors' details}

'Division of Paediatric Rheumatology and Immunology, University Hospital Centre Zagreb, Zagreb, Croatia. ${ }^{2}$ Departement of Ophtalmology, University Hospital Centre Zagreb, Zagreb, Croatia.

Published: 17 September 2014

doi:10.1186/1546-0096-12-S1-P236

Cite this article as: Frkovic et al.: Effects of anti-TNF therapy on ophtalmological complications in children with rheumatic diseases. Pediatric Rheumatology 2014 12(Suppl 1):P236. 